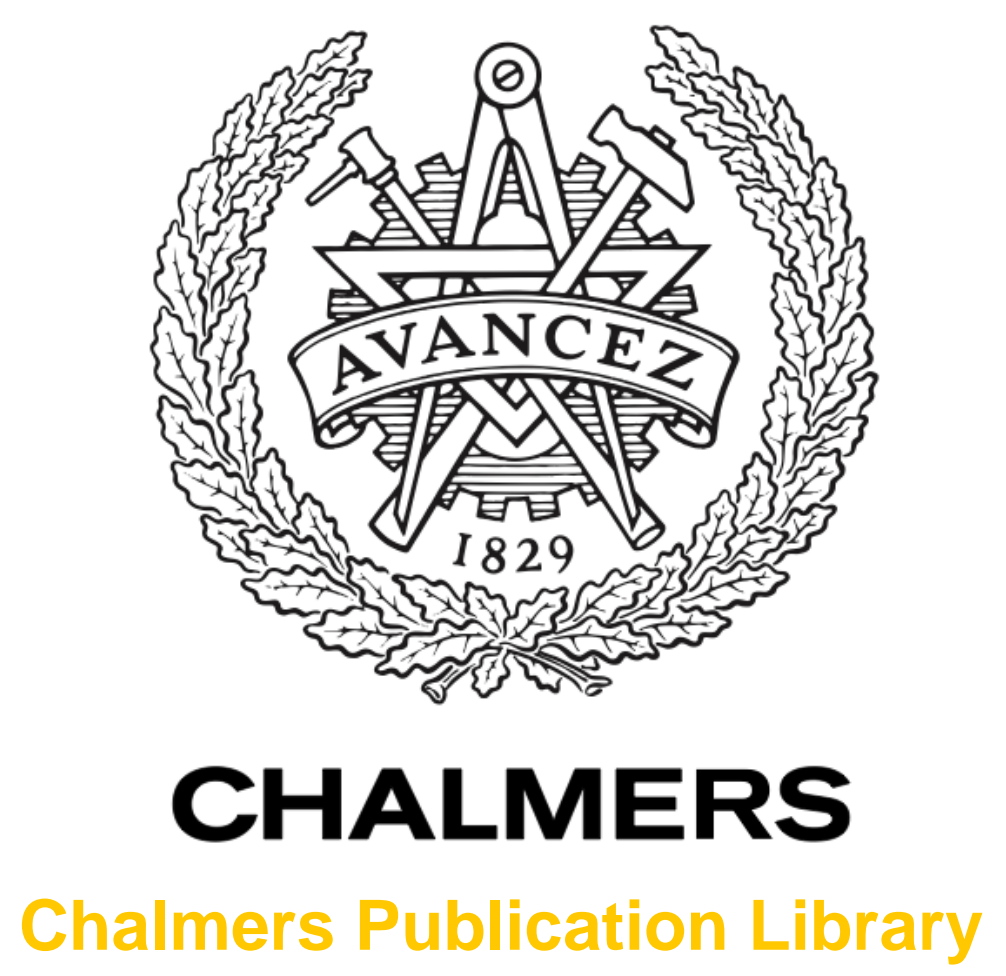

\title{
On Hybrid ARQ and Quantized CSI Feedback Schemes in Quasi-Static Fading Channels
}

This document has been downloaded from Chalmers Publication Library (CPL). It is the author's version of a work that was accepted for publication in:

IEEE transaction on communications (ISSN: 0090-6778)

Citation for the published paper:

Makki, B. ; Eriksson, T. (2012) "On Hybrid ARQ and Quantized CSI Feedback Schemes in Quasi-Static Fading Channels". IEEE transaction on communications, vol. 60(4), pp. 986997.

http://dx.doi.org/10.1109/TCOMM.2012.022912.11

Downloaded from: http://publications.lib.chalmers.se/publication/127073

Notice: Changes introduced as a result of publishing processes such as copy-editing and formatting may not be reflected in this document. For a definitive version of this work, please refer to the published source. Please note that access to the published version might require a subscription. 


\title{
On Hybrid ARQ and Quantized CSI Feedback Schemes in Quasi-Static Fading Channels
}

\author{
Behrooz Makki and Thomas Eriksson \\ Email: behrooz.makki@chalmers.se, thomase@chalmers.se
}

\begin{abstract}
Recently, substantial attention has been paid to increase the achievable rates of wireless networks using different kinds of limited channel quality information feedback. Hybrid automatic repeat request (ARQ) and quantized channel state information (CSI) feedback are two well-known approaches applied by experts to provide the limited channel quality information at the transmitter. Considering quasi-static fading channels, this paper aims to provide some comparisons between the performance of these methods from different points of view. The paper first investigates the power- and outage-limited performance of hybrid ARQ and quantized CSI schemes under short- and long-term power constraints. Then, 1) the feedback signaling load, 2) robustness and 3) the complexity of these schemes are compared under short-term transmission power constraint. Both INcremental Redundancy (INR) and Repetition Time Diversity (RTD) approaches are considered for hybrid ARQ feedback. Finally, approximate low-complexity solutions are presented for power allocation in the INR ARQ-based scheme under long-term transmission power constraint. Analytical and numerical results demonstrate the equivalency or the superiority of these approaches in different circumstances.
\end{abstract}

\section{A. Backgrounds and motivation}

\section{INTRODUCTION}

Adaptive resource allocation techniques are useful tools to increase the transmission efficiency and reliability of wireless networks [1]-[6]. The main idea behind these schemes is to adapt some transmission parameters, such as the transmitter rate and power, based on the channel quality. Therefore, it is essential to have good estimates of the channel gain/SNR at both end-points of the communication link. Motivated by the separate transmission of a training sequence [7], the channel state estimation at the receiver is relatively simple and incurs negligible loss in the transmission rate, particularly when the channel experiences slow variations. On the other hand, due to the signaling load caused by reporting the channel information, assuming perfect channel knowledge at the transmitter is an overly optimistic assumption, which does not 
match with reality. This is the main motivation for the present limited channel quality information feedback systems such as UMTS/WCDMA ${ }^{1}[8]$, WiMAX ${ }^{2}$ [9] and this paper as well.

There are two different approaches, namely, quantized channel state information (CSI) feedback [10][13] and automatic repeat request (ARQ) [14]-[21], providing imperfect channel status information at the transmitter. In a general quantized CSI feedback scheme, the transmitter is provided with some imperfect channel quality information obtained by channel gain quantization at the receiver. This rough information is exploited by the transmitter to select the codewords power and rate such that the system performance is optimized. Throughput, average rate and outage probability are some yardsticks that might be considered as optimization criterion.

$\mathrm{ARQ}$, on the other hand, is a well-known approach applied in today's networks to increase the transmission reliability in the absence of transmitter CSI. In a general ARQ approach, the transmitter considers some initial transmission rate and power with no pre-knowledge about the channel quality. Then, with the help of ARQ, the decoding status at the receiver will be reported back to the transmitter via one bit feedback. The feedback indicates successful decoding of the received signal by an acknowledgement (ACK) bit and failed decoding by a negative acknowledgement (NACK) bit. Based on the received feedback, it is decided by the transmitter whether to retransmit the data or to move on to the next codeword. There are a number of ARQ protocols, such as Repetition Time Diversity (RTD) and INcremental Redundancy (INR) [14]-[16], mainly differing in the retransmission and message decoding procedures.

In many applications, ARQ is used in a passive manner where it is treated only as a binary messagedecoding indicator. That is, the ARQ information is not fully exploited by the transmitter, as different system modules such as channel coding and modulation work as there is no ARQ feedback. However, as shown by, e.g., [14]-[16], [22] and this paper, the ARQ can be utilized in an active manner helping the transmitter to optimize the transmission parameters. In this way, roughly speaking, the CSI and ARQ feedback schemes can be considered as techniques increasing the system throughput by high (low)-rate transmission in high (low) channel quality conditions.

Along with the average rate which characterizes the long-term limits of transmission efficiency, outage

\footnotetext{
${ }^{1}$ Universal Mobile Telecommunication System/Wideband Code Division Multiple Access.

${ }^{2}$ Worldwide Interoperability for Microwave Access.
} 
probability, defined as the probability of the event that the transmitted data is not decodable by the receiver [23]-[25], is another quality-of-service parameter that demonstrates the reliability of a communication network [19], [23]-[31]. While one of the main motivations of ARQ feedback is to minimize the outage probability [19], [25], [26], recent studies have emphasized the positive effect of quantized CSI feedback in reduction of this metric as well [2], [27]-[31]. Therefore, it is interesting to consider the outage as an additional constraint in the rate optimization problem.

\section{B. Related works}

With respect to other related works, the present study mainly roots from the following papers. Following [10], Liu et al., [32] first found the average rate of quasi-static fading channels in the presence of noisefree quantized channel signal-to-noise-ratio (SNR) information at the transmitter and perfect knowledge at the receiver. In their work, in order to have error-free data transmission, the channel SNR is assumed to be constant, equal to its worst case $^{3}$ within each quantization interval ${ }^{4}$. Later, Kim and Skoglund [33] showed that the worst case is, in fact, the best value which can be considered in each quantization region, maximizing the total achievable rate. Further, considering noisy feedback channels, Ekbatani et al., [13], [34] studied the effect of feedback channel noise on the average rate of the quasi-static fading channel.

Implementing noise-free ARQ feedback, on the other hand, [14] and [15] demonstrated the effect of power allocation on the performance of different ARQ protocols. These works were later extended by Shen et al., [16] where they showed that ${ }^{5}$ for any given fading distribution, the maximum average rate of a quasi-static channel in the presence of $M$ bits INR ARQ feedback is equal to the one obtained with CSI feedback quantizing the fading distribution into $N$ regions if 1) the feedback channel is noise-free, 2) shortterm power allocation is considered and 3) $M+1=N$. Finally, considering different power allocation strategies, we extended the results of [16] to the case where both CSI and INR ARQ are simultaneously available for channel quality information feedback [22].

\footnotetext{
${ }^{3}$ Consequently, we denote it "the worst case approach."

${ }^{4}$ In fact, considering a limited number of quantization regions, there will always be a positive outage probability, as the channel may fall in deep fading conditions [33].

${ }^{5}$ [16] has proved the statement for $2^{N}$ CSI-based quantization regions while the extension to any number of quantization regions is straightforward.
} 


\section{Contributions}

The aim of this paper is to present some comparisons between the two well-known hybrid ARQ and quantized CSI feedback approaches providing partial channel quality information at the transmitter. Comparisons are done in different aspects; Section III of the paper focuses on the case where, while the feedback channel noise is negligible, there is an outage limitation on the forward channel data transmission. In this case, we first demonstrate the general average rate maximization problem in the presence of hybrid INR ARQ feedback. Then, we study the problem in more details under two different short- and long-term power constraints. Finally, the same is done for the quantized CSI feedback approach which is followed by some discussions about the equivalency of these methods. To be more clear, considering different feedback schemes, we focus on the following optimization problem

$$
\begin{aligned}
& \max \bar{R} \\
& \text { subject to } \bar{P} \leq P \& \bar{\pi} \leq \pi_{\text {outage }}
\end{aligned}
$$

in which the average rate $\bar{R} \doteq E(R(g))$ is defined as the expectation on $R(g)$, i.e., the successful transmission rate when the channel state is $g$, e.g., [35]. Moreover, $\bar{P}$ is the average transmission power limited to some value $P$ and $\bar{\pi}$ represents the outage probability constrained to be less than $\pi_{\text {outage. }}{ }^{6}$ Furthermore, we present an approximate low-complexity solution for optimal power allocation in the INR ARQ-based data transmission scheme under long-term transmission power constraint.

The second part of the paper studies the feedback load ${ }^{7}$ of the quantized CSI and INR ARQ schemes under different circumstances (Section IV). The results show the superiority of the quantized CSI approach which, for a given feedback signaling load, leads to higher (or equal) forward channel rates. Then, considering one bit feedback, Section V studies the robustness of these schemes in noisy feedback channel conditions. Finally, all results are restudied for the case where RTD approach is implemented for hybrid ARQ feedback and some discussions on the implementation costs and complexities of the considered schemes are presented (Section VI).

\footnotetext{
${ }^{6}$ With perfect CSI at the receiver, on which we focus, the receiver knows whether the transmitted data in each block is decodable or in outage. Therefore, the codewords are either supported error-free or lost. That is, while the unreliable packets are disregarded by the receiver, there will be no transmission errors from this perspective. Consequently, the outage probability constraint considered in this paper is not the symbol (or bit) error probability but can be interpreted as a quality-of-service requirement guaranteeing successful data transmission in a percentage of the time slots. This scenario is normally studied under the topic of receiver-aware outage constraint, e.g., [23], [25], [36].

${ }^{7}$ Here, feedback load is defined as the average number of bits considered for imperfect channel quality information feedback.
} 
In summary, the paper proves the following analytical points:

- The worst case condition: Implementing noise-free quantized CSI feedback, the maximum outagelimited average rate of the channel is obtained when the channel gain is considered to be its lowest value in each quantization interval except the first one (Lemma 1).

- Equivalency: Under noise-free feedback channel assumption and for any outage probability constraint, the optimal outage-limited average rate of the channel with 1) $M$ bits INR ARQ feedback and 2) CSI feedback quantizing the fading distribution into $N$ regions are the same if short-term power allocation is implemented and $M+1=N$ (Theorem 1).

- Extreme outage conditions: Under short-term power constraint, the channel average rate with $N$ CSIbased quantization regions (or $N-1$ INR ARQ feedback bits) and an extremely hard outage probability constraint tends to the same average rate as the case with $N-1$ CSI-based quantization regions (or $N-2$ INR ARQ feedback bits) and no outage probability constraint (Corollary 1).

- Feedback load: Whether outage-limited or not, the same average rate is achieved with less quantized CSI feedback load in comparison to the considered ARQ schemes if 1) the feedback is error-free and 2) a short-term power constraint is considered (Theorem 2).

- Robustness: For any feedback channel bit error probability, 1 bit INR ARQ feedback leads to the same average rate as 1 bit quantized CSI feedback if short-term power allocation is considered (Theorem $3)$.

- Complexity: With the same packeting complexity, quantized CSI approach outperforms the considered hybrid ARQ schemes. Also, INR ARQ wins in competition with RTD in all considered aspects, although it leads to higher implementation complexities (Theorem 4).

\section{Channel MODEL}

As illustrated in Fig.1, we consider a scalar quasi-static fading channel in which the power-limited input message $X, E|X|^{2} \leq P$, multiplied by the random variable $h$ is summed with an independent and identically distributed (iid) complex Gaussian noise $Z \sim \mathcal{C N}\left(0, N_{0}\right)$ resulting in the output

$$
Y=h X+Z
$$

Let us define $g=|h|^{2}$ as the channel gain random variable. The channel gain remains constant for a duration, generally determined by the channel coherence time, and then changes independently according to the fading probability density function (pdf) $f_{G}(g)$. This period, normally called fading block, is supposed 
to be much larger than the length of the codewords containing $K$ nats information ${ }^{8}$. Further, with no loss of generality, we consider $N_{0}=1$.

It is assumed that there is perfect instantaneous knowledge about the channel gain at the receiver, which is an acceptable assumption under quasi-static condition [16], [33]. Also, as in most previous related papers [14]-[16], [22], [33], [34], the partial channel quality information is assumed to be delivered at the transmitter delay-free. Again, this is an acceptable assumption under long fading block conditions. Finally, we focus on infinite backlogged systems where there is an infinite amount of information to be transmitted and so the communication is continuous [37]. In this way, multiple packets, each one having, e.g., several ARQ rounds, are sent within one coherent period. When the channel is good, many packets can be sent within the coherent period, while only few can be transmitted within the same period for bad channels. Therefore, each fading state has the same number of channel uses, and the empirical channel distribution matches the true one. Consequently, as discussed in [16, Section IV], we can consider the channel average rate as a valid performance yardstick. Further, as each transmission experiences an AWGN channel, all results are restricted to Gaussian input distributions. This is a good model of stationary or slow-moving users such as Wireless Local Area Networks (WLANs) [38]. Particularly, since long-block-length capacityapproaching codes can be implemented in such systems, the results can provide realistic insight about the performance bounds of the considered schemes.

Ultimate system performances: Considering different levels of channel quality information available at the transmitter, the average rate of such a channel has been studied in many reports; having full knowledge, it is well accepted, e.g., [16], [33], that the maximum achievable rate can be calculated by ${ }^{9}$

$$
R_{\text {Full }}=\int_{0}^{\infty} f_{G}(g) \log (1+g P(g)) \mathrm{d} g
$$

where $P(g)$ is the optimal power allocation function maximizing the average rate based on the power constraint. On the other hand, with no information about the channel gain realization $g$, a fixed value $g^{*}$ is selected and data transmission is done at rate $r_{\mathrm{No}}^{*}=\log \left(1+g^{*} P\right)$. The data is successfully decoded if

\footnotetext{
${ }^{8}$ All results are presented in natural logarithm basis. Also, in all simulations results the average rate is presented in nats-per-channel-use (npcu).

${ }^{9}$ In an AWGN channel with constant gain $g$ and input power $P$, the maximum rate is obtained by $\log (1+g P)[16]$, [33].
} 
$g \geq g^{*}=\frac{e^{r_{\mathrm{N}}^{*}-1}}{P}$. Consequently, the expected rate is obtained by

$$
R_{\mathrm{No}}=r_{\mathrm{No}}^{*} \operatorname{Pr}\left\{g \geq \frac{e^{r_{\mathrm{No}}^{*}}-1}{P}\right\}=r_{\mathrm{No}}^{*}\left(1-F_{G}\left(\frac{e^{r_{\mathrm{No}}^{*}}-1}{P}\right)\right)
$$

in which $F_{G}($.$) is the gain cumulative distribution function (cdf). Setting \frac{\partial R_{\mathrm{No}}}{\partial r_{\mathrm{No}}^{*}}=0$ yields a necessary condition for a $r_{\mathrm{No}}^{*}$ to be optimal in the sense of average rate ${ }^{10}$.

\section{Outage-Limited CHANNEL AVERAGE RATE}

In this section, we address the rate optimization problem under a noise-free feedback channel assumption when there is an outage limitation on data transmission. The results are obtained for both INR ARQ and CSI feedback approaches under different power allocation constraints.

\section{A. INR ARQ feedback}

As one of the best hybrid ARQ feedback schemes, reaching the highest average rates [16], we mainly focus on the INcremental Redundancy (INR) ARQ protocol where, at each retransmission round, the transmitter sends new redundancy bits and the receiver combines them. In this way, considering $M+1$ maximum allowable ARQ rounds, i.e., maximum $M$ bits ARQ feedback, $K$ information nats is encoded into a codeword of length $T^{(M+1)}$. Then, the codeword is punctured into $M+1$ sub-codewords with powers $P_{m}$ and strictly decreasing rates

$$
R_{m}=\frac{K}{\sum_{k=1}^{m} T_{k}}, m=1, \ldots, M+1 .
$$

Here, $T_{m}$ and $R_{m}$ are the channel uses and the equivalent transmission rate in the $m$-th time slot, respectively. Moreover, $T^{(m)}=\sum_{k=1}^{m} T_{k}$ denotes the total number of channel uses at the end of the $m$-th slot.

At the end of each (re)transmission, 1 bit ARQ feedback is sent back which informs the transmitter about the message decoding status. (Re)transmission continues a maximum of $M+1$ times, in practice determined by the system delay requirements, where finally the data is transmitted at rate $R_{M+1}=\frac{K}{T^{(M+1)}}$ with no ARQ feedback. Outage occurs if the channel instantaneous gain can not support $R_{M+1}$.

Let us define $S_{m}$ as the event that the transmitted data is correctly decoded by the receiver at the $m$-th slot. This event happens if and only if the previous (re)transmissions have been unsuccessful and reducing the rate to $R_{m}$ the receiver can decode the message. Therefore, the average rate and the outage probability can be calculated by

$$
\bar{R}^{\mathrm{INR}}=\sum_{m=1}^{M+1} R_{m} \operatorname{Pr}\left\{\bar{S}_{1}, \ldots, \bar{S}_{m-1}, S_{m}\right\}
$$

\footnotetext{
${ }^{10}$ In order to study more about this rate optimization problem, the readers are referred to [33], where the Karush-Kuhn-Tucker condition is derived.
} 
and

$$
\bar{\pi}^{\mathrm{INR}}=\operatorname{Pr}\left\{\bar{S}_{1}, \ldots, \bar{S}_{M+1}\right\}
$$

respectively, where $\bar{S}_{m}$ is defined as the complement of the event $S_{m}$ and $S_{0}=\emptyset$. Implementing random coding and typical set-based decoding, the results of [23], [39, chapter 15] can be used where $\operatorname{Pr}\left\{S_{m}\right\}$ is simplified to the Time Division Multiple Access (TDMA)-type equation ${ }^{11}$

$$
\operatorname{Pr}\left\{S_{m}\right\}=\operatorname{Pr}\left\{\sum_{k=1}^{m} \frac{T_{k}}{\sum_{j=1}^{m} T_{j}} \log \left(1+g P_{k}\right) \geq R_{m}\right\} .
$$

Note that, based on (4), we have

$$
\frac{T_{k}}{\sum_{j=1}^{m} T_{j}}=R_{m}\left(\frac{1}{R_{k}}-\frac{1}{R_{k-1}}\right)
$$

and so $\operatorname{Pr}\left\{S_{m}\right\}$ is found as a function of $R_{k}$ 's.

The optimal transmission powers $P_{k}$, maximizing the outage- and power-limited average rate, are determined based on the power constraint; with unequal power allocation, the actual average power up to the end of different ARQ rounds is a discrete random variable given by ${ }^{12}$

$$
P(g)=P^{(m)} \text { if } \bar{S}_{1}, \ldots, \bar{S}_{m-1}, S_{m}, P^{(m)}=\frac{\sum_{k=1}^{m} P_{k} T_{k}}{\sum_{k=1}^{m} T_{k}}=R_{m} \sum_{k=1}^{m} P_{k}\left(\frac{1}{R_{k}}-\frac{1}{R_{k-1}}\right)
$$

where $P^{(m)}$ is the average power up to end of the $m$-th slot. Therefore, the average transmission power is obtained by

$$
\bar{P}^{\mathrm{INR}}=\sum_{m=1}^{M+1} P^{(m)} \operatorname{Pr}\left\{\bar{S}_{1}, \ldots, \bar{S}_{m-1}, S_{m}\right\}+P^{(M+1)} \operatorname{Pr}\left\{\bar{S}_{1}, \ldots, \bar{S}_{M+1}\right\} .
$$

In this perspective, considering $\bar{P}^{\mathrm{INR}} \leq P$ and $\bar{\pi}^{\mathrm{INR}} \leq \pi_{\text {outage }}$ as the transmission power and the outage probability constraints, respectively, the general power- and outage-limited rate optimization problem can be stated as

$$
\begin{aligned}
& \bar{R}_{\max }^{\mathrm{INR}}= \max _{\left\{P_{k}, R_{k}, k=1, \ldots, M+1\right\}} \bar{R}^{\mathrm{INR}} \\
& \text { subject to } \bar{P}^{\mathrm{INR}} \leq P \& \bar{\pi}^{\mathrm{INR}} \leq \pi_{\text {outage }}
\end{aligned}
$$

which is a complex non-convex optimization problem even in its simplest cases [16], [33]. In the following, we solve (11) under two different interpretations of the power constraint.

\footnotetext{
${ }^{11}$ As mentioned in Section II, the results are obtained under the assumption that the fading value $g$ is constant over all ARQ (re)transmissions.

${ }^{12}$ Note that the transmission rates and powers are fixed after optimization.
} 
1) Short-term power constraint: Due to, e.g., hardware or complexity limitations, there are cases where, independently of the feedback index, the power allocated can not exceed a maximum value $P$ [16], [22], [33], [34]. In this case, as the transmission rate of AWGN channels is an increasing function of the SNR [40], [41], the optimal powers maximizing the average rate are obtained by $P_{k}=P, \forall k=1, \ldots, M+1$, normally denoted short-term power allocation. Therefore, based on (4) and (5), the achievable rate is a random variable given by

$$
R(g)= \begin{cases}R_{m+1} & \text { if }\left\{R_{m+1} \leq \log (1+g P)<R_{m}\right\}, m=0, \ldots, M \\ 0 & \text { if } R_{M+1}>\log (1+g P)\end{cases}
$$

where $R_{0} \doteq \infty$. Consequently, the average rate is found as

$$
\bar{R}^{\mathrm{INR}}=\sum_{m=0}^{M} R_{m+1}\left(F_{G}\left(u_{m}\right)-F_{G}\left(u_{m+1}\right)\right), u_{m} \doteq \frac{e^{R_{m}}-1}{P}
$$

and the outage probability is simplified to

$$
\bar{\pi}^{\mathrm{INR}}=F_{G}\left(u_{M+1}\right) .
$$

Inserting (13) and (14) in (11), the rate optimization problem can be solved numerically (Fig. 3 and 7). Note that, in contrast to the transmission power, the channel average rate is not a monotonic function of the outage probability. Consequently, its constraint can not be considered as an equality and so, although implementable, gradient-based algorithms are not efficient in this case. In order to tackle this problem, we propose an iterative algorithm illustrated in Algorithm 1.

The proposed algorithm has been shown to be efficient in complex non-convex optimization problems dealing with local minima issues, although, depending on the number of optimization parameters, may be slow in convergence [42]. Finally, note that, with proper modifications, the proposed algorithm can be used for other optimization problems as well.

2) Long-term power constraint: Equation (13) provides the channel average rate under the assumption that the transmit power is constant during the entire $M+1$ transmissions. Intuitively, using optimal power allocation among the (re)transmissions the transmission efficiency can be improved. In this case, (11) should be solved in its most general case which, as mentioned before, leads to a complex optimization problem. In the following, we first study the simplest case of (11) where, using 1 bit ARQ feedback, the effect of power allocation on the average rate of the channel is investigated both analytically and numerically. Later, we present an approximate solution for optimal power allocation under low SNR regime and with arbitrary number of INR-based retransmissions. 
Considering (7)-(10) and $M=1$ bit INR ARQ feedback, the average transmission power, i.e., (10), can be rewritten as

$$
\begin{aligned}
\bar{P}^{\mathrm{INR}} & =P_{1} \operatorname{Pr}\left\{S_{1}\right\}+\frac{P_{1} T_{1}+P_{2} T_{2}}{T_{1}+T_{2}}\left(1-\operatorname{Pr}\left\{S_{1}\right\}\right) \\
& \stackrel{(a)}{=} P_{1} \operatorname{Pr}\left\{S_{1}\right\}+\frac{P_{1} R_{2}+P_{2}\left(R_{1}-R_{2}\right)}{R_{1}}\left(1-\operatorname{Pr}\left\{S_{1}\right\}\right)
\end{aligned}
$$

in which (a) is based on (4) and, from (7), $\operatorname{Pr}\left\{S_{1}\right\}$ is determined by $P\left\{S_{1}\right\}=1-F_{G}\left(\frac{e^{R_{1}}-1}{P_{1}}\right)$. Moreover, using (4), (5) and (7), the average rate of the channel can be calculated as

$$
\begin{aligned}
& \bar{R}^{\mathrm{INR}}=R_{1} \operatorname{Pr}\left\{\log \left(1+g P_{1}\right) \geq R_{1}\right\}+R_{2} \operatorname{Pr}\left\{\log \left(1+g P_{1}\right)<R_{1} \& \frac{T_{1}}{T_{1}+T_{2}} \log \left(1+g P_{1}\right)\right. \\
& \left.+\frac{T_{2}}{T_{1}+T_{2}} \log \left(1+g P_{2}\right) \geq R_{2}\right\} \\
& =R_{1}\left(1-F_{G}\left(\frac{e^{R_{1}}-1}{P_{1}}\right)\right)+R_{2}\left(F_{G}\left(\frac{e^{R_{1}}-1}{P_{1}}\right)-F_{G}(\chi)\right) \text {, } \\
& \chi \doteq \underset{g}{\arg }\left\{y(g)=R_{2}\right\}, y(g)=\frac{R_{2}}{R_{1}} \log \left(1+g P_{1}\right)+\frac{R_{1}-R_{2}}{R_{1}} \log \left(1+g P_{2}\right) .
\end{aligned}
$$

Note that, as $R_{1} \geq R_{2}$, it is easy to show that $y(0) \leq R_{2} \leq y\left(\frac{e^{R_{1}}-1}{P_{1}}\right)$ and $y(g)$ is a monotonic function of $g$. Hence, $\chi$ is unique for any given $R_{1}, R_{2}, P_{1}, P_{2}$. Also, the outage probability is obtained as $\bar{\pi}^{\mathrm{INR}}=F_{G}(\chi)$. In this way, the rate optimization problem (11) is changed into

$$
\begin{aligned}
\bar{R}_{\max }^{\mathrm{INR}}= & \max _{\left\{P_{1}, P_{2}, R_{1}, R_{2}\right\}} R_{1}\left(1-F_{G}\left(\frac{e^{R_{1}}-1}{P_{1}}\right)\right)+R_{2}\left(F_{G}\left(\frac{e^{R_{1}}-1}{P_{1}}\right)-F_{G}(\chi)\right) \\
& \text { subject to } P_{1} \operatorname{Pr}\left\{\mathrm{S}_{1}\right\}+\frac{P_{1} R_{2}+P_{2}\left(R_{1}-R_{2}\right)}{R_{1}}\left(1-\operatorname{Pr}\left\{S_{1}\right\}\right) \leq P \& F_{G}(\chi) \leq \pi_{\text {outage }}
\end{aligned}
$$

which, with proper modifications in algorithm 1, can be solved numerically (See Fig. 4).

Finally, in order to reduce the problem complexity, we propose an approximate solution for (11); In general, it is difficult to find the probabilities $\operatorname{Pr}\left\{S_{m}\right\}$, particularly for large $M$. However, by using (4) and $\log (1+x) \approx x$ under low SNR assumption, (7) can be approximated by

$$
\operatorname{Pr}\left\{S_{m}\right\} \approx \operatorname{Pr}\left\{g \sum_{k=1}^{m} \frac{T_{k} P_{k}}{\sum_{j=1}^{m} T_{j}} \geq R_{m}\right\} \stackrel{(b)}{=} 1-F_{G}\left(z_{m}\right), z_{m}=\frac{R_{m}}{P^{(m)}}
$$

where (b) is based on (9). Consequently, the rate optimization problem (11) is simplified to

$$
\begin{aligned}
& \bar{R}_{\max }^{\mathrm{INR}}=\max _{\left\{P_{m}, R_{m}, m=1, \ldots, M+1\right\}} \sum_{m=1}^{M+1} R_{m}\left(F_{G}\left(z_{m-1}\right)-F_{G}\left(z_{m}\right)\right), z_{0}=\infty \\
& \text { subject to } \sum_{m=1}^{M+1} P^{(m)}\left(F_{G}\left(z_{m-1}\right)-F_{G}\left(z_{m}\right)\right)+P^{(M+1)} F_{G}\left(z_{M+1}\right) \leq P, F_{G}\left(z_{M+1}\right) \leq \pi_{\text {outage }}
\end{aligned}
$$

which is much less complex, in comparison to (11). Note that implementing $\log (1+x) \approx \log (x)$ the optimal transmission powers can be obtained at high SNRs. However, as the effect of optimal power allocation diminishes at high input powers, see e.g., [14]-[16], [33], [34], we do not discuss it any further. 


\section{B. Quantized CSI feedback}

Considering CSI feedback quantizing the fading pdf into $N$ regions, an encoding function

$$
C(g)=c_{i}, g \in G_{i}=\left[g_{i-1}, g_{i}\right), i=1, \ldots, N, g_{0}=0, g_{N}=\infty
$$

is implemented by the receiver and the symbol $c_{i}$ is sent back to the transmitter [32]-[34]. Getting $c_{i}$, due to the quasi-static fading assumption, the channel gain is assumed to be some fixed value $g_{i}^{*} \in\left[g_{i-1}, g_{i}\right)$ and the data is transmitted at rate $r_{i}^{*}=\log \left(1+g_{i}^{*} Q_{i}\right)$ where $Q_{i}$ is the power allocated to the $i$-th input distribution. If the instantaneous gain realization supports the rate, i.e., $g \geq g_{i}^{*}=\frac{e^{r_{i}^{*}}-1}{Q_{i}}$, the transmitted data is successfully decoded, otherwise outage occurs. Therefore, for a given quantization output $c_{i}$, the outage probability $\pi_{i}$ and the expected rate are

$$
\pi_{i}=\operatorname{Pr}\left\{\text { outage } \mid C(g)=c_{i}\right\}=\frac{1}{p_{i}}\left[F_{G}\left(\frac{e^{r_{i}^{*}}-1}{Q_{i}}\right)-F_{G}\left(g_{i-1}\right)\right]
$$

and

$$
\bar{r}_{i}=\frac{1}{p_{i}}\left[F_{G}\left(g_{i}\right)-F_{G}\left(\frac{e^{r_{i}^{*}}-1}{Q_{i}}\right)\right] r_{i}^{*},
$$

respectively. Here, $p_{i}=\int_{g_{i-1}}^{g_{i}} f_{G}(g) \mathrm{d} g$ is the probability of the gain belonging to the $i$-th region, i.e., $G_{i}$. Therefore, the channel average rate is determined as

$$
\bar{R}^{\mathrm{CSI}}=\sum_{i=1}^{N} p_{i} \bar{r}_{i}=\sum_{i=1}^{N}\left[F_{G}\left(g_{i}\right)-F_{G}\left(\frac{e^{r_{i}^{*}}-1}{Q_{i}}\right)\right] r_{i}^{*}
$$

and the outage probability would be

$$
\bar{\pi}^{\mathrm{CSI}}=\sum_{i=1}^{N} p_{i} \pi_{i}=\sum_{i=1}^{N}\left[F_{G}\left(\frac{e^{r_{i}^{*}}-1}{Q_{i}}\right)-F_{G}\left(g_{i-1}\right)\right] .
$$

Finally, the average transmission power is simply found as $\bar{P}^{\mathrm{CSI}}=\sum_{i=1}^{N} p_{i} Q_{i}$.

In order to maximize the average rate with an outage probability and an average transmission power constraint, i.e., solving

$$
\begin{aligned}
& \max \bar{R}^{\mathrm{CSI}} \\
& \text { subject to } \bar{P}^{\mathrm{CSI}} \leq P \& \bar{\pi}^{\mathrm{CSI}} \leq \pi_{\text {outage }}
\end{aligned}
$$

we use the Lagrangian criterion

$$
\begin{aligned}
& R_{\max }^{\mathrm{CSI}}=\max _{\alpha, g_{i}, g_{i}^{*}, Q_{i}, i=1, \ldots, N} \phi\left(\alpha, g_{1}, \ldots, g_{N}, g_{1}^{*}, \ldots, g_{N}^{*}, Q_{1}, \ldots, Q_{N}\right) \\
& \phi\left(\alpha, g_{1}, \ldots, g_{N}, g_{1}^{*}, \ldots, g_{N}^{*}, Q_{1}, \ldots, Q_{N}\right)=\sum_{i=1}^{N}\left[F_{G}\left(g_{i}\right)-F_{G}\left(\frac{r_{i}^{*}-1}{Q_{i}}\right)\right] r_{i}^{*} \\
& +\lambda \sum_{i=1}^{N} p_{i} Q_{i}+\rho_{\alpha} \sum_{i=1}^{N}\left[F_{G}\left(\frac{e^{r_{i}^{*}}-1}{Q_{i}}\right)-F_{G}\left(g_{i-1}\right)\right],
\end{aligned}
$$


where $\lambda$ and $\rho_{\alpha}$ are the Lagrange multiplier coefficients determined based on the constraints $\bar{P}^{\text {CSI }}=P$ and $\bar{\pi}^{\mathrm{CSI}}=\alpha \leq \pi_{\text {outage }}$. Kim and Skoglund [33] have previously shown that with no outage probability constraint the maximum average rate is obtained by considering the channel gain to be equal to its worst case within each region, i.e., $g_{i}^{*}=g_{i-1}, i>1$. The following lemma extends this result to the case when the data transmission is constrained to have limited outage probability.

Lemma 1: (The worst case condition) Under noise-free feedback channel assumption, the optimal outageand power-limited average rate of the channel with CSI feedback quantizing the fading distribution into $N$ regions is obtained by the worst case approach.

Proof: Taking the derivative of $\phi$, in (26), with respect to $g_{i} \in\left[g_{i}^{*}, g_{i+1}^{*}\right], i \geq 1$ leads to

$$
\frac{\partial \phi}{\partial g_{i}}=f_{G}\left(g_{i}\right)\left\{r_{i}^{*}+\lambda\left(Q_{i}-Q_{i+1}\right)-\rho_{\alpha}\right\}
$$

whose sign does not depend on $g_{i}$. Hence, for any value of $\alpha$, the average rate is found as a monotonic function of $g_{i}$ and its maximum is obtained by either $g_{i}=g_{i}^{*}$ or $g_{i}=g_{i+1}^{*}$. However, selecting $g_{i}=g_{i}^{*}$ will eliminate the $i$-th rate term of (23) and thereby increase the outage probability, which is obviously suboptimal. Therefore, it is concluded that for any given outage probability constraint, i.e., $\rho_{\alpha}$, the optimal average rate is obtained by $g_{i}^{*}=g_{i-1}, i>1$.

In this way, the channel average rate can be rewritten as

$$
\bar{R}^{\mathrm{CSI}}=\sum_{i=2}^{N} p_{i} \log \left(1+g_{i-1} Q_{i}\right)+\left(F_{G}\left(g_{1}\right)-F_{G}\left(g_{1}^{*}\right)\right) \log \left(1+g_{1}^{*} Q_{1}\right)
$$

and the outage probability constraint is simplified to $F_{G}\left(g_{1}^{*}\right)=\alpha \leq \pi_{\text {outage }}$. Moreover, setting $\frac{\partial \phi}{\partial Q_{i}}=0$, the optimal transmission powers, in terms of (25), are obtained by the water-filling [23], [33] equations

$$
Q_{i}= \begin{cases}\left\lceil\frac{F_{G}\left(g_{1}\right)-F_{G}\left(g_{1}^{*}\right)}{-\lambda p_{1}}-\frac{1}{g_{1}^{*}}\right\rceil^{+}, & i=1 \\ \left\lceil\frac{-1}{\lambda}-\frac{1}{g_{i-1}}\right]^{+}, & i>1\end{cases}
$$

where $\lceil x\rceil^{+} \doteq \max (0, x)$. Also, the parameters $g_{1}^{*}$ and $g_{i}$ are optimized by appropriate modifications in algorithm 1. (For simulation results, see Fig.5 and 7). Finally, the following theorem demonstrates the equivalency of the INR ARQ and the quantized CSI feedback schemes under outage-limited conditions.

Theorem 1: (Equivalency) Under a noise-free feedback channel assumption and for any outage probability constraint $\bar{\pi} \leq \pi_{\text {outage }}$, the same outage-limited average rate is obtained by $M$ bits INR ARQ feedback and CSI feedback quantizing the fading distribution into $N$ regions if 1) short-term power allocation is implemented and 2) $M+1=N$. 
Proof: Using (13) and (14), the fixed-power outage-limited average rate optimization problem of the channel with $M$ bits INR ARQ feedback, i.e., (11), is rephrased as

$$
\begin{aligned}
\bar{R}_{\max }^{\mathrm{ARQ}}= & \max _{\alpha, R_{m}, \forall m}\left(\sum_{m=1}^{M+1} R_{m} \operatorname{Pr}\left\{R_{m} \leq \log (1+g P)<R_{m-1}\right\}\right) \\
& \text { subject to } F_{G}\left(\frac{e^{R_{M+1}-1}}{P}\right)=\alpha<\pi_{\text {outage }}
\end{aligned}
$$

in which $R_{0} \doteq \infty$. On the other hand, considering CSI feedback with $N$ quantization regions, short-term power constraint and Lemma 1, (25) and (28) can be used to show that the optimal outage-limited average rate of the channel is found by

$$
\begin{aligned}
& \bar{R}_{\max }^{\mathrm{CSI}}=\max _{\alpha, \beta_{m}, \forall m}\left(\sum_{m=1}^{N} \beta_{m} \operatorname{Pr}\left\{\frac{e^{\beta_{m}}-1}{P} \leq g<\frac{e^{\beta_{m+1}}-1}{P}\right\}\right) \\
& \quad \text { subject to } F_{G}\left(\frac{e^{\beta_{1}-1}}{P}\right)=\alpha<\pi_{\text {outage }} \\
& \beta_{m}= \begin{cases}\log \left(1+g_{1}^{*} P\right) & m=1 \\
\log \left(1+g_{m-1} P\right) & m=2, \ldots, N \\
\infty & m=N+1\end{cases}
\end{aligned}
$$

Comparing (30) and (31) under $M+1=N$ condition, the equivalency of the two methods is obvious. In this case, we have $R_{m}=\beta_{M+2-m}, m=0, \ldots, M+1$. Finally, note that, removing the outage probability constraint, i.e., $\pi_{\text {outage }}=1$, the theorem is simplified to [16, Lemma 3].

Corollary 1: Let $\bar{R}_{N}\left(\pi_{\text {outage }}\right)$ be the maximum short-term power-limited average rate of the channel with $N$ quantization regions (or equivalently, with $N-1$ INR ARQ feedback bits) under an outage probability constraint $\bar{\pi} \leq \pi_{\text {outage }}$. Then, $\lim _{\pi_{\text {outage } \rightarrow 0}} \bar{R}_{N}\left(\pi_{\text {outage }}\right)=\bar{R}_{N-1}(1)$. In words, the average rate for the case when the outage probability tends to 0 is equal to the average rate with no outage constraint, with one less region in the quantizer (one less ARQ retransmission round).

Proof: Letting $\pi_{\text {outage }} \rightarrow 0$, the outage probability constraint $F_{G}\left(g_{1}^{*}\right) \leq \pi_{\text {outage }}$ implies $g_{1}^{*} \rightarrow 0$. Therefore, based on (28) under short-term power constraint ${ }_{N} Q_{n}=P$, we have

$$
\lim _{\pi_{\text {outage } \rightarrow 0}} \bar{R}_{N}\left(\pi_{\text {outage }}\right)=\max _{g_{1}, \ldots, g_{N}}\left\{\sum_{n=2}^{N}\left(F_{G}\left(g_{n}\right)-F_{G}\left(g_{n-1}\right)\right) \log \left(1+g_{n-1} P\right)\right\} .
$$

However, (32) is the same as the outage-unlimited average rate maximization problem

$$
\bar{R}_{N-1}(1)=\max _{\widehat{g}_{1}, \ldots, \widehat{g}_{N-1}, \widehat{g}_{1}^{*}}\left\{\sum_{n=2}^{N-1}\left(F_{G}\left(\widehat{g}_{n}\right)-F_{G}\left(\widehat{g}_{n-1}\right)\right) \log \left(1+\widehat{g}_{n-1} P\right)+\left(F_{G}\left(\widehat{g}_{1}\right)-F_{G}\left(\widehat{g}_{1}^{*}\right)\right) \log \left(1+\widehat{g}_{1}^{*} P\right)\right\}
$$

in which the optimization parameters are renamed as $\widehat{g}_{1}^{*}=g_{1}, \widehat{g}_{n}=g_{n+1}, n \geq 1$.

This corollary simply means that under extremely hard outage probability constraints (almost) no data is transmitted when the channel is in deep fading conditions. Therefore, the average rate is obtained by 
summation of the $n \geq 2$ regions expected transmission rates which, based on Lemma 1 , are always decodable at the receiver. Considering the INR ARQ approach, on the other hand, the arguments indicate that under hard outage probability constraints the transmission rates are selected such that in the last retransmission round the equivalent transmission rate is extremely low, and the data is decoded with probability of (almost) 1. Finally, this result is further investigated through Fig.7, as stated in the following.

\section{FEEDBACK LOAD}

Along with the system complexity and commercial issues, one of the most important aspects that affects the system performance is the feedback load caused by reporting the channel quality information [1], [3][6]. In general, considering this issue as one of the optimization constraints will lead to a totally different rate maximization problem where it will change the feedback coding, particularly under a noisy feedback assumption. However, the following theorem shows that, under a noise-free feedback channel assumption and for uniform power allocation, the same average rate is obtained with less (or equal) CSI feedback load in comparison to INR ARQ ${ }^{13}$.

Theorem 2: (Feedback load) Whether outage-limited or not, the same average rate is achieved with less (or equal) noise-free quantized CSI feedback load in comparison to INR ARQ.

Proof: Utilizing maximum $M$ bits INR ARQ feedback, the feedback load is obtained by

$$
\bar{l}^{\mathrm{INR}}=\sum_{m=1}^{M} m \operatorname{Pr}\left\{\bar{S}_{1}, \ldots, \bar{S}_{m-1}, S_{m}\right\}+M \operatorname{Pr}\left\{\bar{S}_{1}, \ldots, \bar{S}_{m-1}, \bar{S}_{M}\right\}
$$

which, using (7) under short-term power constraint, can be written as

$$
\begin{aligned}
l^{\mathrm{INR}} & =\sum_{m=1}^{M} m \operatorname{Pr}\left\{R_{m} \leq \log (1+g P)<R_{m-1}\right\}+M \operatorname{Pr}\left\{0 \leq \log (1+g P)<R_{M}\right\} \\
& =\sum_{m=1}^{M+1} l_{m}^{\mathrm{INR}} \operatorname{Pr}\left\{\mu_{m} \leq g<\mu_{m-1}\right\} \\
l_{m}^{\mathrm{INR}} & =\left\{\begin{array}{l}
m, m=1, \ldots, M \\
M, m=M+1
\end{array}, \mu_{m}= \begin{cases}\infty & m=0 \\
\frac{e^{R_{m}-1}}{P} & m=1 \ldots M \\
0 & m=M+1\end{cases} \right.
\end{aligned}
$$

Here, considering $F_{G}\left(\frac{e^{R_{M+1}}-1}{P}\right)=\alpha<\pi_{\text {outage }}$ as the outage probability constraint, the optimal values of $\mu_{m}$ 's are obtained by (30). Using the CSI-based quantization boundaries $0=g_{0}<g_{1}<g_{2} \ldots<g_{N}=\infty$, on the other hand, the feedback load of the transmission system can be formulated as

\footnotetext{
${ }^{13}$ It is worth noting that the arguments of the paper are valid only for the considered channel quality feedback schemes and conclusions are not necessarily valid for other quantized CSI or hybrid ARQ approaches.
} 


$$
\bar{l}^{\mathrm{CSI}}=\sum_{m=1}^{N} l_{m}^{\mathrm{CSI}} \operatorname{Pr}\left\{g_{m-1} \leq g<g_{m}\right\}
$$

in which $l_{m}^{\text {CSI }}$ is the length of the codeword used for encoding the $m$-th quantization region symbol, i.e., $c_{m}$ in (20). The optimal quantization boundaries are determined based on (31). However, considering $M+1=N$ and the optimal values of $\mu_{m}$ 's and $g_{m}$ 's, Theorem 1 implies $\mu_{m}=g_{M+1-m}, m=0, \ldots, M+1$ and $R_{M+1}=\beta_{1}=\log \left(1+g_{1}^{*} P\right)$. That is, using optimal ARQ or CSI feedback, the sets of points $\left\{\mu_{m}\right\}$ and $\left\{g_{m}\right\}$ partition the fading distribution into the same nonoverlapping regions and the probability terms of both (34) and (35) can be replaced by the set of $\left\{p_{i} \mid i=1 \ldots N, p_{i}=\int_{g_{i-1}}^{g_{i}} f_{G}(g) \mathrm{d} g\right\}$. In this way, the statement

$$
\bar{l}^{\mathrm{CSI}}=\sum_{m=1}^{N} l_{m}^{\mathrm{CSI}} p_{m} \leq \sum_{m=1}^{N} l_{m}^{\mathrm{INR}} p_{M+2-m}=\bar{l}^{\mathrm{INR}}
$$

becomes obvious where, while the code lengths $l_{m}^{\mathrm{INR}}$ are fixed and not necessarily optimal, we can use optimal coding techniques, e.g., Huffman coding [43], to find the optimal lengths $l_{m}^{\text {CSI }}$. These code lengths, that are determined based on the probabilities $p_{i}, i=1 \ldots N$, lead to minimum feedback load while the forward channel average rate is kept the same as with $M$ bits INR ARQ feedback. Note that, although the values of $R_{M+1}$ and $g_{1}^{*}$ do not directly appear in (34) and (35), respectively, they will change the other boundaries based on the outage probability constraint. However, as stated in Theorem 1, the modifications are the same keeping the probability terms of (34) and (35) equivalent. Finally, since the case with no outage probability constraint is an special case of the general outage-limited problem, the proof is valid in that case as well.

\section{Robustness}

In practice, the feedback signals reach the transmitter through a communication link experiencing different levels of noise and feedback channel fading. Hence, it is probable to receive erroneous signals at the transmitter which, if not handled suitably, can degrade the system performance severely and make it even worse than an open-loop system. Therefore, it is interesting to study the channel performance under noisy feedback conditions. However, as using multi-bits feedback the forward channel performance of the quantized CSI-based scheme in noisy conditions is highly dependent on the feedback coding and detection [13], [34], we focus on the case of 1 bit feedback which requires no specific feedback coding. The solution to this case can give valuable insights in other cases as well.

Here, it is assumed that the transmitter is unaware of the presence of feedback channel noise and, consequently, it trusts the received feedback signals. Therefore, the transmission rates are selected such 
that Theorem 1 holds. That is, introducing the noise in the feedback channel, we are interested in studying the robustness of ARQ and CSI feedback approaches in working at realistic conditions. Under such a condition, Theorem 3 shows the equivalency of the quantized CSI and INR ARQ feedback under shortterm power constraint.

Theorem 3: (Robustness) For any feedback channel bit error probability, 1 bit INR ARQ feedback leads to the same average rate as 1 bit quantized CSI feedback if short-term power allocation is considered.

Proof: Let $p_{e}$ be the feedback channel bit error probability. As illustrated in Fig.2a, implementing 1 bit quantized CSI feedback the fading pdf is divided into two regions and the transmission rates are selected based on the worst case condition (Lemma 1). In this way, provided that the transmitter decodes the quantized CSI feedback as $c_{1}\left(\right.$ or $\left.c_{2}\right)$, the data is transmitted at rate $r_{1}^{*}=\log \left(1+g_{1}^{*} P\right)\left(\right.$ or $\left.r_{2}^{*}=\log \left(1+g_{1} P\right)\right)$ which is received if $g>g_{1}^{*}$ ( or $g>g_{1}$ ). Therefore, the average rate is found as

$$
\begin{aligned}
\bar{R}^{\mathrm{CSI}} & =r_{1}^{*} \operatorname{Pr}\left\{g \geq g_{1}^{*} \mid c_{1} \text { is decoded at transmitter }\right\}+r_{2}^{*} \operatorname{Pr}\left\{g \geq g_{1} \mid c_{2} \text { is decoded at transmitter }\right\} \\
& =r_{1}^{*}\left\{\left(F_{G}\left(g_{1}\right)-F_{G}\left(g_{1}^{*}\right)\right)\left(1-p_{e}\right)+\left(1-F_{G}\left(g_{1}\right)\right) p_{e}\right\}+r_{2}^{*}\left(1-F_{G}\left(g_{1}\right)\right)\left(1-p_{e}\right) .
\end{aligned}
$$

Now, consider Fig.2b. Utilizing 1 bit INR ARQ, two cases can happen if the gain instantaneous realization satisfies $g \geq \frac{e^{R_{1}-1}}{P}$; in the cases where the ARQ feedback is correctly decoded at the transmitter, $K$ nats information is successfully transmitted via $T_{1}$ channel uses and the transmission rate would be $R_{1}=\frac{K}{T_{1}}$. On the other hand, if the ARQ feedback is wrongly decoded, while still $K$ nats information is received by the receiver, $T_{2}$ channel uses are wasted for sending more parity bits and so the transmission rate reduces to $R_{2}=\frac{K}{T_{1}+T_{2}}$. Therefore, the average transmission rate obtained in this case would be $\left.\bar{R}^{\mathrm{INR}}\right|_{g \geq \frac{e^{R_{1-1}}}{P}}=R_{1}(1-$ $\left.p_{e}\right)+R_{2} p_{e}$. Further, given that the gain instantaneous realization is within the region $g \in\left[\frac{e^{R_{2}-1}}{P}, \frac{e^{R_{1}-1}}{P}\right)$, the data is successfully decoded if and only if the ARQ feedback bit is correctly received by the transmitter. Consequently, the expected transmission rate in this case is $\left.\bar{R}^{\mathrm{INR}}\right|_{g \in\left[\frac{e^{R_{2}-1}}{P}, \frac{e^{R_{1}-1}}{P}\right)}=\frac{K}{T_{1}+T_{2}}\left(1-p_{e}\right)=R_{2}\left(1-p_{e}\right)$ and the channel average rate is obtained as

$$
\bar{R}^{\mathrm{INR}}=\left(R_{1}\left(1-p_{e}\right)+R_{2} p_{e}\right)\left(1-F_{G}\left(\frac{e^{R_{1}}-1}{P}\right)\right)+R_{2}\left(1-p_{e}\right)\left(F_{G}\left(\frac{e^{R_{1}}-1}{P}\right)-F_{G}\left(\frac{e^{R_{2}}-1}{P}\right)\right) .
$$

Now, based on the fact that, with no knowledge about the feedback channel noise, the transmission rates are optimized such that $R_{2}=r_{1}^{*}$ and $R_{1}=r_{2}^{*}$ (Theorem 1), one can use (37) and (38) to show that $\bar{R}^{\mathrm{INR}}=\bar{R}^{\mathrm{CSI}}$. 
This is an interesting conclusion particularly when we remind that in many applications 1 bit CSI feedback has been shown to be enough to reach (almost) peak performance of the data transmission system [3], [4], [6]. Also, note that using 1 bit feedback leads to the same feedback load in both methods, keeping the comparison fair.

\section{PACKETING COMPLEXITY}

Talking about the system implementation complexity, different practical aspects should be considered. This section presents some discussions on the coding complexity of the quantized CSI and hybrid ARQ schemes as follows.

In contrast to the INR ARQ feedback, the quantized CSI approach is among the fixed-length coding techniques where, regardless of the channel conditions, the length of transmission packets is constant. Moreover, considering INR ARQ, in each retransmission new parity packets should be generated based on the message decoding status. Hence, the quantized CSI is better than the INR ARQ approach in terms of packeting complexity. Adding any code length constraint in the INR ARQ approach will deteriorate its efficiency leading to lower transmission rates, in comparison to the quantized CSI scheme. However, it is interesting to investigate the system performance when utilizing fixed-length-code HARQ techniques, among which the RTD is one of the best ones, e.g., [14]-[16], [25].

Using RTD ARQ feedback, the same packet is retransmitted in each retransmission and the receiver performs maximum ratio combining of all received packets. Therefore, it leads to substantially less packeting complexity, as not only the code lengths are the same in all retransmissions but also no new parity packet needs to be generated in the retransmission rounds. Under such conditions, the transmission parameters are obtained as follows; considering fixed power $P$ in all (re)transmissions ${ }^{14}$, at the $m$-th RTD (re)transmission round the received SNR increases to $m P$ and the effective rate reduces to $\frac{R}{m}$ in which $R$ is the original code rate. Therefore, one can use the results of, e.g., [14]-[16], [25], to show that the achievable rates random variable, i.e., (12), is changed to

$$
R(g)=\left\{\begin{array}{ll}
\frac{R}{m}, & \log (1+(m-1) g P) \leq R<\log (1+m g P) \\
0, & R>\log (1+(M+1) g P)
\end{array} .\right.
$$

In this way, the noise-free average rate, outage probability, feedback load and the average rate with 1 bit noisy feedback, i.e., (13), (14), (33) and (38), are respectively changed to

\footnotetext{
${ }^{14}$ The effect of power allocation on the performance of RTD ARQ scheme can be studied in, e.g., [25].
} 


$$
\begin{gathered}
\bar{R}^{\mathrm{RTD}}=\sum_{m=1}^{M+1} \frac{R}{m}\left(F_{G}\left(\frac{e^{R}-1}{(m-1) P}\right)-F_{G}\left(\frac{e^{R}-1}{m P}\right)\right), \\
\bar{\pi}^{\mathrm{RTD}}=F_{G}\left(\frac{e^{R}-1}{(M+1) P}\right), \\
l^{\mathrm{RTD}}=\sum_{m=1}^{M} m\left(F_{G}\left(\frac{e^{R}-1}{(m-1) P}\right)-F_{G}\left(\frac{e^{R}-1}{m P}\right)\right)+M \times F_{G}\left(\frac{e^{R}-1}{M P}\right)
\end{gathered}
$$

and

$$
\bar{R}^{\mathrm{RTD}}=\left(R\left(1-p_{e}\right)+\frac{R}{2} p_{e}\right)\left(1-F_{G}\left(\frac{e^{R}-1}{P}\right)\right)+\frac{R}{2}\left(1-p_{e}\right)\left(F_{G}\left(\frac{e^{R}-1}{P}\right)-F_{G}\left(\frac{e^{R}-1}{2 P}\right)\right) .
$$

Theorem 4: (INR vs RTD ARQ feedback) Comparing RTD and INR hybrid ARQ feedback schemes under short-term power constraint, the following statements hold:

(I): With the same number of maximum retransmission rounds, INR outperforms RTD in the sense of outage-limited average rate.

(II): With the same feedback load (the average number of retransmissions), the INR ARQ scheme leads to higher forward channel average rates, in comparison to RTD.

(III): For any feedback channel bit error probability, higher rates are obtained by 1 bit INR than by 1 bit RTD hybrid ARQ.

Proof: Here, we only prove part (II) while the other parts can be proven with the same arguments. Let $\tilde{R}$ be the optimal transmission parameter maximizing (40). Then, we set the INR transmission rates such that $\frac{e^{R m}-1}{P}=\frac{e^{\tilde{R}}-1}{m P}$. Note that these values are not necessarily the optimal ones maximizing (13). In this way, the probability terms of (34) and (42) will be the same leading to the same feedback load in the INR and RTD ARQ schemes. Then, as the probability terms of (13) and (40) are equal, the statement (II) is proven if we show that $R_{m}=\log \left(\frac{e^{\tilde{R}}+m-1}{m}\right) \geq \frac{\tilde{R}}{m}$. However, defining the function $h(\tilde{R})=\log \left(\frac{e^{\tilde{R}}+m-1}{m}\right)-\frac{\tilde{R}}{m}$, it is easily found that $h(0)=0$ and $\frac{\partial h}{\partial \tilde{R}} \geq 0$. Therefore, we have $h(\tilde{R}) \geq 0, \forall \tilde{R} \geq 0$, i.e., $R_{m} \geq \frac{\tilde{R}}{m}$. Consequently, from (13) and (40), higher rates are obtained by INR scheme than by RTD ARQ feedback. The statements (I) and (III) can be proven with the same technique.

Finally, because of the equivalency between CSI and INR ARQ, it is obvious that the quantized CSI outperforms the RTD ARQ approach in all considered comparison yardsticks. However, focusing on implementation issues, there are other practical aspects that should be considered as well; Quantized CSI feedback belongs to the physical layer techniques and, in order to exploit its properties, needs some additional designs, particularly at the transmitter [44]. This point increases the system implementation 
costs. ARQ, on the other hand, is a technique in the data link layer which is already provided in most of wireless protocols. Therefore, it needs no additional closed-loop design which introduces it as a cost-efficient approach in the current communication systems.

\section{Simulation RESUlts}

The simulation results are obtained for Rayleigh-fading channels, i.e., $f_{G}(g)=\omega e^{-\omega g}, g \geq 0$, where we set $\omega=1$. Considering no outage probability limitation and short-term power constraint, Fig.3 shows the system average rate in the presence of $M$ bits INR ARQ or, equivalently, quantized CSI feedback scheme with $N=M+1$ quantization regions (Theorem 1). As it can be seen, substantial rate increment is achieved with limited number of feedback bits. Then, Fig.4 demonstrates the effect of one bit INR ARQ feedback on the channel average rate with different power and outage probability constraints. Also, Fig.5 evaluates the effect of different power allocation strategies and outage probability constraint on the average rate of the system utilizing quantized CSI feedback. The figures indicate that while the long-term power allocation increases the transmission rates, its effect diminishes as the transmission power increases. Then, Fig.6 studies the system average rate in the presence of RTD ARQ feedback. Finally, considering short-term power constraint $P=2$, Fig.7 verifies the effect of outage probability constraint on the system performance when utilizing different quantized CSI or hybrid ARQ feedback schemes. While the figure indicates the validity of Corollary 1 for quantized CSI or INR ARQ feedback, the RTD-based achievable rates converge to zero under hard outage probability constraints. Also, the figure emphasizes the superiority of INR ARQ (or quantized CSI) scheme in comparison with RTD ARQ protocol.

\section{CONCLUSION}

This paper presented some comparisons between the performance of data transmission systems utilizing standard hybrid ARQ and quantized CSI feedback schemes. Comparisons were done in different aspects. Particularly, we showed that:

- Under a short-term power constraint and with proper selection of quantization regions and the ARQ feedback bits, the same outage-limited average rate is achieved by the quantized CSI and the INR hybrid ARQ schemes.

- With the same forward channel average rate, the quantized CSI scheme outperforms the INR protocol in terms of feedback load. 
- Considering noisy feedback channels, the same average rate is obtained by 1-bit quantized CSI and 1-bit INR-based hybrid ARQ feedback.

- The quantized CSI and the INR hybrid ARQ approaches outperform the RTD hybrid ARQ protocol in terms of average rate, feedback load and robustness.

Finally, selecting the best approach is not easy since the decision depends on several parameters such as complexity and commercial issues.

\section{REFERENCES}

[1] B. Makki and T. Eriksson, "Efficient channel quality feedback signaling using transform coding and bit allocation," in VTC, May 2010, accepted.

[2] _ _ "Data transmission in the presence of channel state feedback and outage probability constraint," in VTC, Sept. 2010, accepted.

[3] T. Eriksson and T. Ottosson, "Compression of feedback for adaptive transmission and scheduling," Proc. of the IEEE, vol. 95, no. 12, pp. 2314-2321, Dec. 2007.

[4] —_ "Compression of feedback in adaptive OFDM-based systems using scheduling," IEEE Commun. Lett., vol. 11, no. 11, pp. 859-861, Nov. 2007.

[5] J. C. Roh and B. D. Rao, "Transmit beamforming in multiple-antenna systems with finite rate feedback: a VQ-based approach," IEEE Trans. on Info. Theory, vol. 52, no. 3, pp. 1101-1112, March 2006.

[6] S. Sanayei and A. Nosratinia, "Exploiting multiuser diversity with only 1-bit feedback," in WCNC, vol. 2, March 2005, pp. 978-983.

[7] E. Biglieri, J. Proakis, and S. Shamai, "Fading channels: information-theoretic and communications aspects," IEEE Trans. on Info. Theory, vol. 44, no. 6, pp. 2619-2692, Oct. 1998.

[8] "Technical specification group radio access network, physical layer procedures," 3rd Generation Partnership Project, Tech. Spec. 3GPP ts 25.214 V3.10.0, 2002-2003.

[9] "Physical and medium access control layers for combined fixed and mobile operation in licensed bands," iEEE Std. Std 802.16e-2005, 2005.

[10] A. J. Goldsmith and P. P. Varaiya, "Capacity of fading channels with channel side information," IEEE Trans. on Info. Theory, vol. 43, no. 6, pp. 1986-1992, Nov. 1997.

[11] S. Tatikonda and S. Mitter, “The capacity of channels with feedback,” IEEE Trans. on Info. Theory, vol. 55, no. 1, pp. 323-349, Jan. 2009.

[12] N. C. Martins and T. Weissman, "Coding for additive white noise channels with feedback corrupted by quantization or bounded noise," IEEE Trans. on Info. Theory, vol. 54, no. 9, pp. 4274-4282, Sept. 2008.

[13] S. Ekbatani, F. Etemadi, and H. Jafarkhani, "Throughput maximization over slowly fading channels using quantized and erroneous feedback," IEEE Trans. on Commun., vol. 57, no. 9, pp. 2528-2533, Sept. 2009.

[14] D. Tuninetti, "Transmitter channel state information and repetition protocols in block fading channels," in ITW, Sept. 2007, pp. 505-510.

[15] J. Perret and D. Tuninetti, "Repetition protocols for block fading channels that combine transmission requests and state information," in ICC, May 2008, pp. 1297-1301.

[16] C. Shen, T. Liu, and M. P. Fitz, "On the average rate performance of hybrid-ARQ in quasi-static fading channels," IEEE Trans. on Commun., vol. 57, no. 11, pp. 3339-3352, Nov. 2009. 
[17] H. E. Gamal, G. Caire, and M. O. Damen, “The diversity-multiplexing-delay tradeoff in MIMO ARQ channels," in ISIT, Sept. 2005, pp. $1823-1827$.

[18] N. Gopalakrishnan and S. Gelfand, "Rate selection algorithms for IR hybrid ARQ," in IEEE Sarnoff Symposium, April 2008, pp. 1-6.

[19] P. Wu and N. Jindal, "Performance of hybrid-ARQ in block-fading channels: A fixed outage probability analysis," IEEE Trans. on Commun., vol. 58, no. 4, pp. 1129-1141, April 2010.

[20] K. D. Nguyen, L. K. Rasmussen, A. Guillen i Fabregas, and N. Letzepis, "MIMO ARQ systems with multi-level feedback," in ISIT, July 2009, pp. 254-258.

[21] — "Rate-diversity-delay tradeoff for ARQ systems over MIMO block-fading channels," in AusCTW, Feb. 2009, pp. 116-121.

[22] B. Makki and T. Eriksson, "On the average rate of quasi-static fading channels with ARQ and CSI feedback," IEEE Commun. Lett., vol. 14 , no. 9, pp. 806-808, Sept. 2010 .

[23] G. Caire, G. Taricco, and E. Biglieri, “Optimum power control over fading channels,” IEEE Trans. on Info. Theory, vol. 45, no. 5, pp. 1468-1489, July 1999.

[24] E. Biglieri, G. Caire, and G. Taricco, "Limiting performance of block-fading channels with multiple antennas," IEEE Trans. on Info. Theory, vol. 47, no. 4, pp. 1273-1289, May 2001.

[25] W. Su, S. Lee, D. A. Pados, and J. D. Matyjas, "The optimal transmission power per round for hybrid-ARQ Rayleigh fading links," in ICC, May 2010, pp. 1-5.

[26] A. Chuang, A. Guillen i Fabregas, L. K. Rasmussen, and I. B. Collings, "Optimal throughput-diversity-delay tradeoff in MIMO ARQ block-fading channels," IEEE Trans. on Info. Theory, vol. 54, no. 9, pp. 3968-3986, sept. 2008.

[27] V. Annapureddy, D. Marathe, T. R. Ramya, and S. Bhashyam, "Outage probability of multiple-input single-output (MISO) systems with delayed feedback," IEEE Trans. on Commun., vol. 57, no. 2, pp. 319 -326, Feb. 2009.

[28] X. Zhang, F. Chen, and W. Wang, "Outage probability study of multiuser diversity in MIMO transmit antenna selection systems," IEEE Sig. Proc. Lett., vol. 14, no. 3, pp. 161-164, March 2007.

[29] Y. Xie, C. N. Georghiades, and A. Arapostathis, "Minimum outage probability transmission with imperfect feedback for MISO fading channels," IEEE Trans. on Wireless Commun., vol. 4, no. 3, pp. 1084-1091, May 2005.

[30] A. A. Dowhuszko, G. Corral-Briones, J. Hamalainen, and R. Wichman, "Outage probability analysis of practical multiuser scheduling schemes with limited feedback," in VTC, April 2007, pp. 1036-1040.

[31] J. Chen and K.-K. Wong, "Communication with causal CSI and controlled information outage," IEEE Trans. on Wireless Commun., vol. 8, no. 5, pp. 2221-2229, May 2009.

[32] X. Liu, H. Yang, W. Guo, and D. Yang, "Capacity of fading channels with quantized channel side information," IEICE Trans. on Commun., vol. E89-B(2), pp. 590-593, 2006.

[33] T. T. Kim and M. Skoglund, "On the expected rate of slowly fading channels with quantized side information," IEEE Trans. on Commun., vol. 55, no. 4, pp. 820-829, April 2007.

[34] S. Ekbatani, F. Etemadi, and H. Jafarkhani, "Transmission over slowly fading channels using unreliable quantized feedback," in $D C C$, March 2007, pp. 353-362.

[35] M. Effros, A. Goldsmith, and Y. Liang, "Capacity definitions of general channels with receiver side information," in ISIT, June 2007, pp. 921-925.

[36] X. Kang, R. Zhang, Y. C. Liang, and H. K. Garg, "Optimal power allocation strategies for fading cognitive radio channels with primary user outage constraint," IEEE J. on Sel. Areas in Commun., vol. 29, no. 2, pp. 374-383, 2011. 
[37] L. Badia, M. Rossi, and M. Zorzi, "SR ARQ packet delay statistics on markov channels in the presence of variable arrival rate," IEEE Trans. on Wireless Commun., vol. 5, no. 7, pp. 1639-1644, July 2006.

[38] R. Narasimhan, "Individual outage rate regions for fading multiple access channels," in ISIT, June 2007, pp. 1571-1575.

[39] T. M. Cover and J. A. Thomas, Elements of Information Theory. New York: Wiley Interscience, 1992.

[40] A. Lozano, A. M. Tulino, and S. Verdu, "Optimum power allocation for parallel Gaussian channels with arbitrary input distributions," IEEE Trans. on Info. Theory, vol. 52, no. 7, pp. 3033-3051, July 2006.

[41] D. Guo, S. Shamai, and S. Verdu, "Mutual information and minimum mean-square error in Gaussian channels," IEEE Trans. on Info. Theory, vol. 51, no. 4, pp. 1261-1282, April 2005.

[42] B. Makki, M. Noori Hosseini, S. A. Seyyedsalehi, and N. Sadati, "Unaligned training for voice conversion based on a local nonlinear principal component analysis approach," Neural comp. and appl., vol. 19, no. 3, pp. 437-444, Nov. 2009.

[43] K. Sayood, Introduction to Data Compression. Morgan Kaufmann Publishers, 3rd ed., 2006.

[44] M. Vu, "Exploiting transmit channel side information in MIMO wireless systems," Ph.D. dissertation, Stanford University, Stanford, CA, 2006. 